\title{
INTEGRATION OF BACILLUS SUBTILIS WITH SOME ESSENTIAL PLANT OILS FOR THE CONTROL OF ONION WHITE ROT.
}

\author{
MAHMOUD, E.Y., M.M. IBRAHIM, FATMA A. MOSTAFA and \\ ZEINAB N. HUSSIEN
}

Plant Pathology Res. Ins., Agric. Res. Center, Giza., Egypt

(Manuscript received 29 May 2016)

\begin{abstract}
$\checkmark 1$ reenhouse and field experiments were conducted in 2013/2014 and 2014/2015 to study the effect of Bacillus subtilis, when applied as soil treatment in combination with different concentrations of essential plant oils as dipping treatment on onion white rot infection. Oils of cumin, cardamom and thyme were tested at five concentrations $(0.25 \%, 0.50 \%, 1 \%, 2 \%$, and $4 \%$, on the linear growth of Sclerotium cepivorum isolates. Cumin oil had the strongest inhibitory effect on linear growth followed by thyme. Reduction in linear growth increased by increasing concentration of the tested plant oil. Under artificial and natural infection conditions in greenhouse and field trials, $B$. subtilis combined with different concentrations of essential plant oils were significantly effective in reducing incidence of onion white rot, compared with untreated control. Also, treatments increased fresh and dry weight under greenhouse conditions as well as increased onion bulb yield in field trials. Generally, cumin oils followed by thyme with $B$. subtilis gave the highest percentage of apparent healthy plants as well as the highest efficacy in reducing onion white rots compared to other treatments except fungicides treatment (Trust EW 25). The superior treatments were obtained by cumin at $2 \%$ with $B$. subtilis and it was the nearest treatment to fungicides in reducing of onion white rots incidence.

Keywords: Sclerotium cepivorum, Bacillus subtilis, bioagent, cumin, thyme, cardamom, biological control, essential plant oils and fungicides
\end{abstract}

\section{INTRODUCTION}

Onion (Allium cepa L.) is the most widely cultivated Allium species in Egypt. Onion production has been significantly reduced in the last years due to white rot disease caused by Sclerotium cepivorum Berk (Khalifa et al. 2013 a \& b). The disease has become widely distributed in Egypt and is considered a limiting factor especially in upper Egypt (Mohamed, 2012). The pathogen produces numerous long-lived small size survival structures (sclerotia) that can last for many years in the soil and serve as a primary source of inoculum (Jones, 2010).

Due to the environmental regulations and the weakness of chemical control, biological control has become more attractive (Cook, 1993). Mishra et al., (2013) 
defined biological control as the reduction of the amount of inoculum of a pathogen accomplished by or through one or more microorganisms. Bacteria, especially plant growth-promoting rhizobacteria (PGPR), were reported to suppress a variety of root and vascular diseases caused by soilborne pathogens. Bacillus was considered as an important member of these bacteria and its application under greenhouse and field conditions, reduced damping-off and root rot and crown rot diseases caused by Rhizoctonia solani, Macrophomina phaseolina and Fusarium oxysporum. (Mahmoud, 2014).

Essential oils are concentrated, hydrophobic liquid containing volatile aromatic compounds. They may provide potential alternatives to the control means currently used because of bioactive chemicals. They have biological activities such as antimicrobial activity, attributed to certain fractions or to sulfur-containing compounds in the aqueous phase, responsible for the aroma and flavor characteristic (ElMoshtohory, 2007).

Volatiles of many spices such as Thyme were proved to be one of the most effective inhibitor of the microbial growth and having a good antibacterial activity with direct contact and also were used as drugs (Leal et al ., 2003). EL-Bastawesy and Mohamed (2005) examined the volatile and non-volatile components of cardamom for their antifungal activity against two strains of spoilage fungi namely $A$. flavus and A.ochraceus. They found that the inhibitory effect of volatile oil against some pathogenic fungi increased as concentration increased. Moreover, Kapoor (2008) found that the cardamom essential oil caused $100 \%$ inhibition of Aspergillus flavus at a $6 \mu$.

Ozcan and Erkmen (2001) studied the antifungal activity of essential oils of 9 spices (cumin, savory, laurel, oregano, basil, seafennel, myrle, pickling herb and mint) at 3 concentrations (1,10 and $15 \%$ ) on yeast (Saccharomyces cerevisia and Candida rugosa) and fungi (Rhizopus oryzae and Aspergillus niger). They stated that, cumin, savory, and oregano had inhibitor effect. Moreover Cuminum cyminum inhibited Candida albicans, Candida rugosa, Saccharomyces cerevisia and Aspergillus niger (Mahmoud and Gomaa, 2015). Also, Akash et al., (2014) stated that, cumin essential oil was able to enhance food products against fungi and aflatoxin contamination and lipid peroxidation.

The present study was conducted to investigate the effect of some plant oils as transplant dipping treatment combined with Bacillus subtilis as soil treatment for the suppression of onion white rot. 


\section{MATERIALS \& METHODS}

The fungal isolates, used throughout this study were previously isolated by the authors from diseased onion and their pathogenic capabilities were determined (Khalifa et al., 2013 b).

\section{Preparation of fungal inoculum:}

The Inoculum of Sclerotium cepivorum was prepared using sorghum - coarse sand - water $(2: 1: 2 \mathrm{v} / \mathrm{v})$ medium. The ingredients were mixed, bottled and autoclaved at 15 pound / sq. inch for $2 \mathrm{~h}$. Each bottle of sterilized medium was inoculated with $5 \mathrm{~mm}$ fungal growth disc obtained from the periphery of 5-day-old culture of the fungus. The inoculated media were incubated at $28^{\circ} \mathrm{C}$ for 15 days before used for soil infestation.

\section{Soil infestation:}

Fungal propagules of $S$. cepivorum were mixed thoroughly with the surface soil of each pot, at the rate of $2 \% \mathrm{w} / \mathrm{w}$, and then covered with a thin layer of sterilized soil. Pots containing infested soil were irrigated and kept for 10 days until sown.

\section{Disease assessment:}

a) The number of plants having typical white rot symptoms was counted after two and four month from planting and their percentage were calculated according to Hovius and Goldman (2004) as follows:

$\%$ White rot $=\frac{\text { Number of plants infested with white rot }}{\text { Total No. of plants }} \times 100$

$\%$ Healthy plants $=\frac{\text { Number of survived healthy plants }}{\text { Total No. of plants }} \times 100$

C) Percent efficacy of treatment in reducing the diseases infection was calculated.

$\%$ Treatment efficiency $=\frac{\text { Control }- \text { Treatment }}{\text { Control }} \times 100$

Relative efficiency of oils to fungicides efficacy $=\frac{\text { Plant oils efficiency }}{\text { Fungicide efficiency }} \mathrm{X} 100$

\section{Source of antagonistic bacteria:}

Known isolate of Bacillus subtilis; Bs1 (El-Hadidy, 2003) was obtained from Culture Collection of the Department of Plant Pathology, Faculty of Agriculure, Ain Shams University, Cairo, Egypt.

\subsection{Preparation of bacterial inoculum:}

The Bacillus isolate was grown on nutrient agar medium, Bacterial suspensions $\left(1 \times 10^{6} \mathrm{cfu} / \mathrm{ml}\right)$ were prepared by dilution plate assay as described by Callan et al., (1990). 


\subsection{Methods of bacterial inoculum application:}

The antagonistic bacteria $\left(10^{6} \mathrm{cfu} / \mathrm{ml}\right)$ were applied as a soil drench after 7 (approximately $10 \mathrm{ml} /$ pore) and 15 days from planting.

\section{Use of plant oils:}

Commercial essential oils of Cumin, Cardamom and thyme were used in the present work. Essential oils used in the study were obtained from Chemical Industrial Development Company (CID), Egypt.

\subsection{Chemical analysis of plant oils tested}

The obtained Essential oils analyzed at the Agricultural Research Center (ARC) Plant Pathology Res. Inst. Biotechnology Unit. Using GC Mass System (Agilent Technologies 5975 Inert XL Mass Selective Detector). As reported by Basyony et al., 1989.

\subsection{Effect of different plant oils on the linear growth of the pathogen in vitro:}

The inhibitory effect of different concentrations of the plant oils namely Cumin, Cardamom and thyme on the in vitro linear growth of $S$. cepivorum isolates were evaluated. Plant oils were diluted in distilled water plus $95 \%$ ethanol $(10 \% \mathrm{v} / \mathrm{v})$ and tween $80(0.5 \% \mathrm{v} / \mathrm{v})$ aseptically to obtain different concentrations namely 0.25 , $0.50,1,2$, and $4 \%$. (were added to a known amount of sterilized PDA and immediately before pouring). After medium solidification the plates were inoculated with $5 \mathrm{~mm}$ disk of each fungal isolates and incubated at $27{ }^{\circ} \mathrm{C}$. Five plates for each particular treatment were used as replicates. Linear growth was observed daily and diameters of fungal colonies in "mm" were recorded when any of control plates with the fungal growth.

\subsection{Methods of plant oils application:}

For studying the effect of three different plant oils, ( $0.5 \%, 1 \%$ and $2 \%$ concentrations were prepared by using $55 \%$ ethanol $(10 \% \mathrm{v} / \mathrm{v})$ and tween $80(0.5 \%$ $\mathrm{v} / \mathrm{v}$ ) (as dispersing agent) and onion transplant were dipped in these preparations for 2 hours then planted.

\section{Greenhouse experiments}

Pots (50 cm-diam) were sterilized by $5.0 \%$ formalin solution for 15 minutes, left to dry for two days to get rid of formalin residues, then filled with soil previously sterilized by formalin solution (5.0\%) for 15 days and left for 15 days. Fungal propagules were added to the potted soil as mentioned before.

Apparently healthy onion transplants of Giza 20 cultivar were dipped for 2 hours in plant oils concentrations, then planted at the rate of 10 transplants per pot. Also, bacterial suspensions were applied as a soil drench at the 7 and 15 days from 
planting (100 ml / pot). Fungicide Trust EW 25\% (Tebuconazole) applied as dippe for $5 \mathrm{~min}$. at the rate of $25 \mathrm{~cm}^{3} / 1$ liter of water and treatment as soil drench after 6 and 12 weeks at the rate of 187.5/100 liter of water. Experiment was replicated for four times. Disease assessment was recorded as previously mentioned. Also, fresh weight of onion plants from each pot of different treatments were recorded directly after harvest as $\mathrm{g} /$ pot and dried in an oven at about $70^{\circ} \mathrm{C}$ for two days to obtain the dry weights.

\section{Field experiments:}

Field experiments were carried out during onion sowing seasons in 2014 and 2015 under naturally infested soil, at El-Gemiza Experimental Station (ARC). Randomized complete block design with four replicates was used and the plot was 3.0 x $3.5 \mathrm{~m}^{2}\left(10.5 \mathrm{~m}^{2}=1 / 400\right.$ feddan). Each plot included 6 rows (each $3.0 \mathrm{~m}$ length and $50 \mathrm{~cm}$ width). Sixty day-old transplants of onion cultivar Giza 20 were planted per each plot at the recommended spacing $10 \mathrm{~cm} \times 10 \mathrm{~cm}$, within each row the first week of December. Onion transplants were planted on the first week of December at approximately 90 plants/row. The recommended agricultural practices and irrigation for onion crop were followed. The experiment was arranged in completely randomized block design with four replicates. Disease assessment recorded as previously mentioned.

\section{Statistical analysis}

The data were statistically analyzed by analysis of variance (ANOVA) using the Statistical Analysis System (SAS Institute, inc, 1996). Means were separation by least significant difference (L.S.D.) Test at $P \leq 0.05$ and $P \leq 0.01$ level.

\section{RESULTS}

\section{Chemical analysis of plant oils tested}

The active components in the tested plant oils (Tables 1,2 and 3) indicate the presence of thirty-three fractions in cumin, seventeen fractions in cardamom and twenty nine fractions in thyme. 
Table 1.The relative concentration of active components in cumin oil

\begin{tabular}{|c|c|c|c|}
\hline Peak & Rt & relative conc. & Compound name \\
\hline 1 & 7.611 & 1.66 & Alpha-pinen \\
\hline 2 & 8.968 & 1.6 & Pinene \\
\hline 3 & 16.418 & 11.75 & Beta-cumic aldehyde \\
\hline 4 & 20.457 & 1.21 & Beta-metha-1,4-dien-7-01 \\
\hline 6 & 21.55 & 2.45 & 1,4-Benzenediamine \\
\hline 7 & 21.865 & 2.5 & Myrtenal \\
\hline 9 & 22.5 & 3.76 & Benzenetganol ,alpha-methyl \\
\hline 10 & 23.433 & 12.78 & Unknown \\
\hline 11 & 23.69 & 1.7 & Benzoxirene \\
\hline 12 & 24.056 & 5.47 & Carotol \\
\hline 13 & 24.583 & 0.72 & 4-pyridinol, acetate (ester) \\
\hline 14 & 24.806 & 1.28 & Daucol \\
\hline 15 & 25.218 & 2.29 & Guaiol \\
\hline 16 & 26.666 & 1.03 & Isopropyl-4-methyl \\
\hline 19 & 27.244 & 2.17 & Phenol,2,5-dimethyl \\
\hline 20 & 27.564 & 1.43 & Coumarin \\
\hline 21 & 27.69 & 1.46 & Benzenetganol ,alpha-methyl \\
\hline 22 & 28.954 & 8.48 & Unknown \\
\hline 23 & 29.269 & 0.6 & Cembrene \\
\hline 24 & 29.63 & 1.37 & 2,5-Dimethylhydroquinone \\
\hline 25 & 29.973 & 4.39 & 1-butenyl-thiophene \\
\hline 26 & 30.682 & 3.12 & Preclathridine A \\
\hline 27 & 31.123 & 3.85 & Phenathrenemethanol \\
\hline 28 & 31.426 & 3.04 & Unknown \\
\hline 29 & 31.649 & 2.07 & 2-pyridylacetamide \\
\hline 30 & 31.85 & 1.9 & Alpha-terpinyl propinate-gamma-terpinene \\
\hline 31 & 32.01 & 1.54 & E,E-Alpha- farnesene \\
\hline 32 & 32.256 & 2.48 & E,E-Alpha- farnesene \\
\hline 33 & 34.219 & 3.95 & 2-hydroxyphenyl \\
\hline \multicolumn{2}{|c|}{ Total } & 92.05 & \\
\hline
\end{tabular}


Table 2.The relative concentration of active components in cardamom oil

\begin{tabular}{|c|c|c|l|}
\hline Peak & Rt & relative conc. & \multicolumn{1}{|c|}{ Compound name } \\
\hline 1 & 7.617 & 2.59 & Alpha-pinene \\
2 & 10.358 & 15.6 & 1,8 -cineole \\
3 & 13.173 & 1.32 & Gamma -terpinene \\
4 & 13.465 & 3.68 & Alpha- Terpinolene \\
5 & 16.787 & 21.6 & Terpinyl acetate \\
6 & 21.064 & 6.5 & 6,11-Dimethyl-2,6,10-dodecatrien-1-01 \\
7 & 21.344 & 2.69 & Alpha-Terpinenyl acetate \\
8 & 21.808 & 2.97 & 4(5)-acetyl-2-(2-propyl)-1H-imidazole \\
9 & 22.048 & 4.77 & Beta-selinene \\
10 & 22.334 & 2.84 & Unknown \\
11 & 22.855 & 5.04 & 1,2-Ethanediol,1,2-dimyrtenyl -(E)-3(10)-caewn-2-ol \\
12 & 23.141 & 7.24 & Nerolidol \\
13 & 23.456 & 6.09 & Unknown \\
14 & 23.616 & 2.73 & Beta-Gurjunene \\
15 & 24.8 & 6.33 & 5-Aminoindazole \\
16 & 25.435 & 1.14 & 2-pentene,2-methyl \\
17 & 25.556 & 2.78 & Farnesol Isomer B \\
\hline \multicolumn{2}{|l}{ Total } & 95.9 & \\
\hline
\end{tabular}

The major components in cumin oil were cumic aldehyde, in thyme oil were 1,8-cineol and phenol 1- methylethyl. While, the major components in cardamom oil were 1,8-cineole and terpinyl acetate.

Table 3.The relative concentration of active components in thyme oil

\begin{tabular}{|c|c|c|l|}
\hline Peak & Rt & relative conc. & Compound name \\
\hline 1 & 6.662 & 0.7 & Alpha -pinene \\
\hline 2 & 7.835 & 1.74 & Sabinene \\
\hline 3 & 8.252 & 1.1 & 2-Beta-pinene \\
\hline 4 & 9.465 & 23.96 & 1,8 -cineol \\
\hline 5 & 10.175 & 1.45 & Gamma-Terpinene \\
\hline 6 & 11.445 & 4.42 & Linalool L \\
\hline 7 & 13.248 & 0.36 & Borneol L \\
\hline 8 & 13.476 & 2.02 & Terpinene-4-01 \\
\hline 9 & 13.894 & 3.72 & Alpha terpineol \\
\hline 10 & 15.096 & 1.21 & Cuminic \\
\hline 11 & 15.227 & 1.3 & Linalyl acetate \\
\hline 12 & 15.473 & 0.69 & Geraniol \\
\hline 13 & 16.292 & 0.99 & Benzyl alcohol \\
\hline 14 & 17.058 & 20.54 & Phenol 1- methylethyl \\
\hline 15 & 17.665 & 14.5 & Aplpha -terpinenyl acetate \\
\hline 16 & 18.077 & 0.21 & Carvacryl acetate \\
\hline 17 & 18.214 & 0.63 & Geranyl acetate \\
\hline 18 & 19.067 & 0.87 & Trans -caryophyllene \\
\hline 19 & 20.034 & 0.16 & Benzoic acid \\
\hline 20 & 20.497 & 0.56 & Tert- butylcatechin \\
\hline 22 & 22.511 & 0.37 & Caryophllene oxide \\
\hline 23 & 24.394 & 0.31 & Apiol \\
\hline 27 & 29.607 & 16.17 & n-hexadecanoic acide \\
\hline 29 & 31.077 & 0.75 & Linolieic acid methyl ester \\
\hline \multicolumn{2}{|l|}{} & 98.73 & \\
\hline & & & \\
\hline
\end{tabular}




\section{Effect of essential plant oils on the linear growth of S. cepivorum:}

This study was conducted to evaluate the effects of plant oils at five concentrations $(0.25 \%, 0.50 \%, 1 \%, 2 \%$ and $4 \%)$ on the linear growth of different $S$. cepivorum isolates isolated from different locations (Beni-Suef, El-Sharkya and ELNobarya) Data in Table (4) clearly showed that, linear growth was significantly reduced by the tested plant oils treatments.

Increasing the concentration of essential plant oils led to an increase in linear growth reduction. Cumin oil was the most effective followed by thyme oil while cardamom oil recorded the least inhibitive of the three isolates tested. (Table 1).

\section{Effect of transplant treatments with plant oils and soil application of $B$.} subtilis on infection with $S$. cepivorum in greenhouse.

\subsection{On onion white rot incidence:}

Data presented in Table (5) showed that $B$. subtilis when applied as a soil drench combined with different concentrations of plant oils as dipping treatment significantly decreased onion white rot incidence in most treatments compared with untreated control.

Table 4. Effect different concentrations of plant oils on the linear growth of studied pathogens

\begin{tabular}{|c|c|c|c|c|}
\hline \multirow{2}{*}{ Plant oils } & \multirow{2}{*}{$\begin{array}{l}\text { Conc. } \\
(\%)\end{array}$} & \multicolumn{3}{|c|}{ Linear growth of S. cepivorum isolates (mm) } \\
\hline & & Beni - Suef & El-Sharkya & EL-Nobarya \\
\hline \multirow{5}{*}{ Thyme } & 0.25 & 6.6 & 6.1 & 4.6 \\
\hline & 0.50 & 4.3 & 3.9 & 4.3 \\
\hline & 1.0 & 3.4 & 3.2 & 4.1 \\
\hline & 2.0 & 3.1 & 2.8 & 3.7 \\
\hline & 4.0 & 2.8 & 2.5 & 3.4 \\
\hline \multirow{5}{*}{ Cardamom } & 0.25 & 7.6 & 6.9 & 5.0 \\
\hline & 0.50 & 4.5 & 4.0 & 5.0 \\
\hline & 1.0 & 3.5 & 3.3 & 4.6 \\
\hline & 2.0 & 3.2 & 3.0 & 4.2 \\
\hline & 4.0 & 3.0 & 2.8 & 3.9 \\
\hline \multirow{5}{*}{ Cumin } & 0.25 & 3.7 & 3.5 & 2.9 \\
\hline & 0.50 & 2.8 & 2.6 & 2.8 \\
\hline & 1.0 & 2.4 & 2.3 & 2.7 \\
\hline & 2.0 & 2.3 & 2.1 & 2.5 \\
\hline & 4.0 & 2.1 & 2.0 & 2.4 \\
\hline \multicolumn{2}{|c|}{ Control } & 9.0 & 9.0 & 9.0 \\
\hline \multicolumn{5}{|l|}{ L.S.D. $1 \%:$} \\
\hline \multirow{3}{*}{\multicolumn{2}{|c|}{ 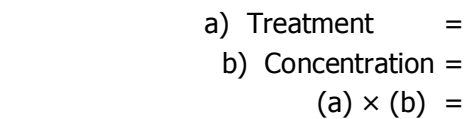 }} & 0.20 & 0.18 & 0.16 \\
\hline & & 0.25 & 0.23 & 0.21 \\
\hline & & 0.36 & 0.32 & 0.29 \\
\hline
\end{tabular}

All tested plant oils at all concentrations showed significantly reduced incidence of onion white rot incidence (\%) compared to non-treated control. Generally, cumin oils followed by thyme at $2 \%$ with $B$. subtilis gave the highest percentage apparent healthy plants as well as the highest efficacy in reducing of 
onion white $\operatorname{rot}(56.0$ and $52 \%)$ compared to other $(72.5$ and70.0\%) treatments except fungicides treatment. The presented data showed that, there is a relationship between plant oils concentrations and their effect on the incidence of onion white rots. (Table 5).

The results obtained also showed that, cumin oil at $2 \%$ with $B$. subtilis was the nearest to fungicides effect (Table 5 ) as it a mentioned to $87.5 \%$ of the fungicides efficacy.

Table 5. Effect of transplant treatment with plant oils and soil application of $B$. subtilis on onion white rot disease under soil infestation with $S$. cepivorum in greenhouse.

\begin{tabular}{|c|c|c|c|c|c|c|}
\hline \multicolumn{2}{|c|}{ Treatments } & \multirow[b]{2}{*}{ Conc. } & \multicolumn{4}{|c|}{ White rot diseases (\%) } \\
\hline Bio-agent & Plant oils & & $\%$ Infection & $\%$ Survival & \% Efficacy & $\begin{array}{c}\text { \%Efficacy to } \\
\text { Fungicides }\end{array}$ \\
\hline \multirow{10}{*}{ 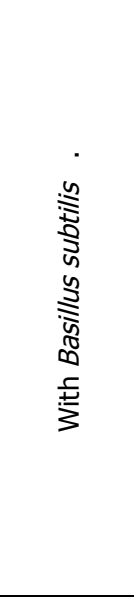 } & & 0.5 & 47.5 & 52.5 & 24.0 & 37.5 \\
\hline & Thyme & 1.0 & 37.5 & 62.5 & 40.0 & 62.5 \\
\hline & & 2.0 & 30.0 & 70.0 & 52.0 & 81.3 \\
\hline & & 0.5 & 40.0 & 60.0 & 36.0 & 56.3 \\
\hline & Cumin & 1.0 & 32.5 & 67.5 & 48.0 & 75.0 \\
\hline & & 2.0 & 27.5 & 72.5 & 56.0 & 87.5 \\
\hline & & 0.5 & 52.5 & 47.5 & 16.0 & 25.0 \\
\hline & Cardamom & 1.0 & 40.0 & 60.0 & 36.0 & 56.3 \\
\hline & & 2.0 & 32.5 & 67.5 & 48.0 & 75.0 \\
\hline & B. subtilis & 0.0 & 60.0 & 40.0 & 4.0 & 6.3 \\
\hline \multirow{10}{*}{ 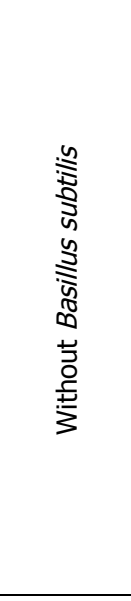 } & & 0.5 & 52.5 & 47.5 & 16.0 & 25.0 \\
\hline & Thyme & 1.0 & 44.0 & 56.0 & 29.6 & 46.3 \\
\hline & & 2.0 & 32.5 & 67.5 & 48.0 & 75.0 \\
\hline & & 0.5 & 47.5 & 52.5 & 24.0 & 37.5 \\
\hline & Cumin & 1.0 & 37.5 & 62.5 & 40.0 & 62.5 \\
\hline & & 2.0 & 30.0 & 70.0 & 52.0 & 81.3 \\
\hline & & 0.5 & 57.5 & 42.5 & 8.0 & 12.5 \\
\hline & Cardamom & 1.0 & 45.0 & 55.0 & 28.0 & 43.8 \\
\hline & & 2.0 & 37.5 & 62.5 & 40.0 & 62.5 \\
\hline & Control & 0.0 & 62.5 & 37.5 & - & - \\
\hline \multicolumn{3}{|c|}{ Fungicides } & 22.5 & 77.5 & 64.0 & 100 \\
\hline
\end{tabular}

\begin{tabular}{|}
$\mid$\begin{tabular}{|c|c|c|c|c|c|c|}
\hline LSD. at $5 \%$ \\
\hline B. subtilis (a) & Plant oils (b) & Conc. (c) & $\mathrm{a} \times \mathrm{b}$ & $\mathrm{a} \times \mathrm{c}$ & $\mathrm{b} \times \mathrm{c}$ & $\mathrm{a} \times \mathrm{b} \times \mathrm{c}$ \\
\hline 3.53 & 5.59 & 4.32 & 7.65 & N.S. & 10.84 & N.S. \\
\hline
\end{tabular}
\end{tabular}




\subsection{On onion fresh and dry weight:}

Results illustrated in table (6) showed that, all tested treatments and their combinations significantly increased onion fresh and dry weight. Fresh and dry weights were highly increased by applying $B$. subtilis with different treatments of plant oils.

Fresh and dry weight were affected significantly by increasing concentrations of plant oils dipping treatments from 0.0 to $2 \%$. The highest increase in fresh and dry weight of onion was obtained by cumin oil at $2 \%$ combined with $B$. subtilis treatment at $94.8 \%$ and $86.7 \%$ respectively and it was the nearest one to fungicides treatment (Table 6).

Table 6. Effect of transplant treatments with plant oils and soil application of $B$. subtilis on onion fresh and dry weight under soil infestation with $S$. cepivorum in greenhouse.

\begin{tabular}{|c|c|c|c|c|c|c|}
\hline \multicolumn{2}{|c|}{ Treatments } & \multirow{2}{*}{ Conc. } & \multirow{2}{*}{$\begin{array}{c}\begin{array}{c}\text { Fresh } \\
\text { weight } / \mathrm{g}\end{array} \\
\end{array}$} & \multirow{2}{*}{ Increase \% } & \multirow{2}{*}{$\begin{array}{c}\text { Dry } \\
\text { weight } / g\end{array}$} & \multirow{2}{*}{ Increase $\%$} \\
\hline Bio-agent & Plant oils & & & & & \\
\hline \multirow{10}{*}{ 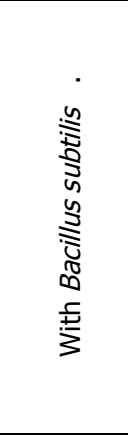 } & \multirow{3}{*}{ Thyme } & 0.5 & 139.2 & 38.8 & 125.0 & 45.4 \\
\hline & & 1.0 & 179.9 & 79.5 & 137.6 & 58.0 \\
\hline & & 2.0 & 187.3 & 86.9 & 150.2 & 70.6 \\
\hline & \multirow{3}{*}{ Cumin } & 0.5 & 174.7 & 74.3 & 132.2 & 52.6 \\
\hline & & 1.0 & 180.1 & 79.7 & 153.7 & 74.1 \\
\hline & & 2.0 & 195.2 & 94.8 & 166.3 & 86.7 \\
\hline & \multirow{3}{*}{ Cardamom } & 0.5 & 130.0 & 29.6 & 107.4 & 27.8 \\
\hline & & 1.0 & 177.2 & 76.8 & 136.8 & 57.2 \\
\hline & & 2.0 & 186.4 & 86.0 & 149.5 & 69.9 \\
\hline & B. subtilis & 0.0 & 128.5 & 28.1 & 115.9 & 36.3 \\
\hline \multirow{10}{*}{ 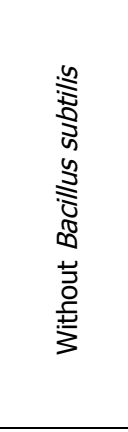 } & \multirow{3}{*}{ Thyme } & 0.5 & 121.6 & 21.2 & 109.2 & 29.6 \\
\hline & & 1.0 & 157.6 & 57.2 & 128.4 & 48.8 \\
\hline & & 2.0 & 184.2 & 83.8 & 141.4 & 61.8 \\
\hline & \multirow{3}{*}{ Cumin } & 0.5 & 154.7 & 54.3 & 132.2 & 52.6 \\
\hline & & 1.0 & 179.5 & 79.1 & 135.9 & 56.3 \\
\hline & & 2.0 & 184.6 & 84.2 & 149.2 & 69.6 \\
\hline & \multirow{3}{*}{ Cardamom } & 0.5 & 115.1 & 14.7 & 103.7 & 24.1 \\
\hline & & 1.0 & 158.2 & 57.8 & 121.2 & 41.6 \\
\hline & & 2.0 & 178.2 & 77.8 & 129.1 & 49.5 \\
\hline & Control & 0.0 & 100.4 & - & 79.6 & - \\
\hline \multicolumn{3}{|c|}{ Fungicides } & 198.1 & 97.7 & 163.3 & 83.7 \\
\hline
\end{tabular}

\begin{tabular}{|c|c|c|c|c|c|c|c|}
\hline LSD. at 5\% & B. subtilis (a) & Plant oils (b) & Conc. (c) & $\mathrm{a} \times \mathrm{b}$ & $\mathrm{a} \times \mathrm{c}$ & $\mathrm{b} \times \mathrm{c}$ & $\mathrm{a} \times \mathrm{b} \times \mathrm{c}$ \\
\hline Fresh weight & 2.15 & 3.44 & 3.44 & 5.26 & 5.26 & 7.84 & 11.49 \\
\hline Dry weight & 2.58 & 4.06 & 4.06 & 6.14 & N.S. & 9.08 & 13.25 \\
\hline
\end{tabular}


3. Effect of transplant treatment with plant oils and soil application of $B$. subtilis under field conditions during seasons 2013/2014 and 2014/2015.

\subsection{On onion white rot:}

Data presented in tables (7\&8) showed that the effect of $B$. subtilis when applied as soil drench combined with different concentrations of plant oils as dipping treatment were significantly effective for reducing white rot disease incidence in two successive seasons 2013/2014 and 2014/2015 compared with untreated control . Also, increasing the concentration of plant oils (from 0.0 to $2 \%$ ) showed more and significant effects in decreasing disease incidence.

Table 7. Effect of transplant treatment with plant oils and soil application of $B$. subtilis on onion white rot incidence under field conditions during season 2013/2014.

\begin{tabular}{|c|c|c|c|c|c|c|}
\hline \multicolumn{2}{|c|}{ Treatments } & \multirow[b]{2}{*}{ Conc. } & \multicolumn{4}{|c|}{ White rot diseases (\%) } \\
\hline $\begin{array}{l}\text { Bio- } \\
\text { agent }\end{array}$ & Plant oils & & $\%$ Infection & \% Survival & \% Efficacy & $\begin{array}{c}\text { \%Efficacy to } \\
\text { Fungicides }\end{array}$ \\
\hline \multirow{10}{*}{ 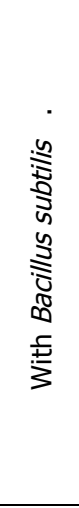 } & \multirow{3}{*}{ Thyme } & 0.5 & 30.4 & 69.6 & 33.9 & 49.5 \\
\hline & & 1.0 & 24.4 & 75.6 & 47.0 & 68.5 \\
\hline & & 2.0 & 20.0 & 80.0 & 56.6 & 82.5 \\
\hline & \multirow{3}{*}{ Cumin } & 0.5 & 23.4 & 76.6 & 49.1 & 71.5 \\
\hline & & 1.0 & 20.9 & 79.1 & 54.6 & 79.6 \\
\hline & & 2.0 & 18.2 & 81.8 & 60.5 & 88.2 \\
\hline & \multirow{3}{*}{ Cardamom } & 0.5 & 33.3 & 66.7 & 27.7 & 40.4 \\
\hline & & 1.0 & 27.1 & 72.9 & 41.1 & 59.9 \\
\hline & & 2.0 & 22.2 & 77.8 & 51.8 & 75.5 \\
\hline & B. subtilis & 0.0 & 37.6 & 62.4 & 18.4 & 26.8 \\
\hline \multirow{10}{*}{ 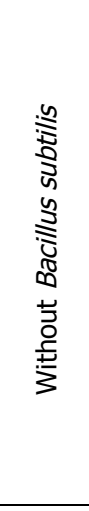 } & \multirow{3}{*}{ Thyme } & 0.5 & 36.3 & 63.7 & 21.2 & 30.9 \\
\hline & & 1.0 & 28.6 & 71.4 & 38.0 & 55.4 \\
\hline & & 2.0 & 24.0 & 76.0 & 48.0 & 69.9 \\
\hline & \multirow{3}{*}{ Cumin } & 0.5 & 27.2 & 72.8 & 40.9 & 59.7 \\
\hline & & 1.0 & 23.4 & 76.6 & 49.2 & 71.8 \\
\hline & & 2.0 & 21.4 & 78.6 & 53.5 & 78.0 \\
\hline & \multirow{3}{*}{ Cardamom } & 0.5 & 37.0 & 63.0 & 19.7 & 28.6 \\
\hline & & 1.0 & 31.1 & 69.0 & 32.6 & 47.5 \\
\hline & & 2.0 & 26.3 & 73.7 & 42.9 & 62.6 \\
\hline & Control & 0.0 & 46.1 & 53.9 & - & - \\
\hline \multicolumn{3}{|c|}{ Fungicides } & 14.5 & 85.5 & 68.6 & 100.0 \\
\hline
\end{tabular}

\begin{tabular}{|c|c|c|c|c|c|c|c|}
\hline LSD. at 5\% & B. subtilis (a) & Plant oils (b) & Conc. (c) & $\mathrm{a} \times \mathrm{b}$ & $\mathrm{a} \times \mathrm{c}$ & $\mathrm{b} \times \mathrm{c}$ & $\mathrm{a} \times \mathrm{b} \times \mathrm{c}$ \\
\hline $2013 / 2014$ & 0.96 & 1.52 & 1.18 & 2.33 & 2.03 & 2.62 & N.S. \\
\hline
\end{tabular}


Generally, cumin oils at $2 \%$ with $B$. subtilis gave the highest percentage of apparent healthy plants as well as the highest efficacy in reducing of onion white rots compared to other treatments except the fungicide treatment

As for the combination, obtained data indicated that the percentage of white rot incidence in two successive seasons were affected significantly by the interaction between $B$. subtilis and plant oils treatments. The results obtained showed also that, B. subtilis + Cumin at $2 \%$ was the nearest to fungicides efficacy in reducing onion white rot (Table 7\&8).

Table 8. Effect of transplant treatment with plant oils and soil application of $B$. subtilis on onion white rot disease under field conditions during season 2014/2015.

\begin{tabular}{|c|c|c|c|c|c|c|}
\hline \multicolumn{2}{|c|}{ Treatments } & \multirow[b]{2}{*}{ Conc. } & \multicolumn{4}{|c|}{ White rot diseases (\%) } \\
\hline $\begin{array}{c}\text { Bio- } \\
\text { agent }\end{array}$ & Plant oils & & $\%$ Infection & \% Survival & \% Efficacy & $\begin{array}{c}\text { \%Efficacy to } \\
\text { Fungicides }\end{array}$ \\
\hline \multirow{10}{*}{ 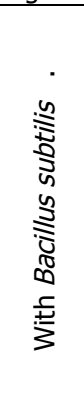 } & \multirow{3}{*}{ Thyme } & 0.5 & 37.6 & 62.4 & 31.2 & 46.3 \\
\hline & & 1.0 & 29.7 & 70.3 & 45.7 & 67.8 \\
\hline & & 2.0 & 23.8 & 76.2 & 56.6 & 83.9 \\
\hline & \multirow{3}{*}{ Cumin } & 0.5 & 31.7 & 68.3 & 42.1 & 62.5 \\
\hline & & 1.0 & 25.7 & 74.3 & 53.0 & 78.6 \\
\hline & & 2.0 & 21.8 & 78.2 & 60.2 & 89.3 \\
\hline & \multirow{3}{*}{ Cardamom } & 0.5 & 41.6 & 58.4 & 24.0 & 35.6 \\
\hline & & 1.0 & 31.7 & 68.3 & 42.1 & 62.5 \\
\hline & & 2.0 & 25.7 & 74.3 & 53.0 & 78.6 \\
\hline & B. subtilis & 0.0 & 49.5 & 50.5 & 9.5 & 14.1 \\
\hline \multirow{10}{*}{ 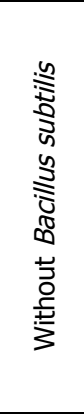 } & \multirow{3}{*}{ Thyme } & 0.5 & 45.5 & 54.5 & 16.8 & 24.9 \\
\hline & & 1.0 & 35.6 & 64.4 & 34.9 & 51.7 \\
\hline & & 2.0 & 25.7 & 74.3 & 53.0 & 78.6 \\
\hline & \multirow{3}{*}{ Cumin } & 0.5 & 37.6 & 62.4 & 31.2 & 46.3 \\
\hline & & 1.0 & 29.7 & 70.3 & 45.7 & 67.8 \\
\hline & & 2.0 & 28.9 & 71.1 & 47.2 & 70.0 \\
\hline & \multirow{3}{*}{ Cardamom } & 0.5 & 47.5 & 52.5 & 13.1 & 19.5 \\
\hline & & 1.0 & 39.1 & 60.9 & 28.4 & 42.2 \\
\hline & & 2.0 & 29.7 & 70.3 & 45.7 & 67.8 \\
\hline & Control & 0.0 & 54.7 & 45.3 & - & - \\
\hline \multicolumn{3}{|c|}{ Fungicides } & 17.8 & 82.2 & 67.4 & 100.0 \\
\hline
\end{tabular}

\begin{tabular}{|c|c|c|c|c|c|c|c|}
\hline LSD. at 5\% & B. subtilis (a) & Plant oils (b) & Conc. (c) & $\mathrm{a} \times \mathrm{b}$ & $\mathrm{a} \times \mathrm{c}$ & $\mathrm{b} \times \mathrm{c}$ & $\mathrm{a} \times \mathrm{b} \times \mathrm{c}$ \\
\hline $2014 / 2015$ & 1.17 & 1.85 & 1.43 & 2.79 & 2.41 & 3.17 & N.S. \\
\hline
\end{tabular}

\subsection{On onion bulb yield:}

Data in table (9) revealed that most of the treatments significantly increased onion bulb yield $\mathrm{kg} / \mathrm{plot}$ in the two seasons. Applying $B$. subtilis with different concentrations of plant oils were more and significantly effective for increasing onion bulb yield than treatments without $B$. subtilis. Also, increasing concentration of plant oils (from 0.0 to $2 \%$ ) was accompanied with higher onion bulb yield. 
The superior results in this regard were obtained by combined treatments of B. subtilis and cumin at $2 \%$ during two growing seasons $2013 / 2014$ and $2014 / 2015$ (Table 9).

Table 9. Effect of transplant treatment with plant oils and soil application of $B$. subtilis on onion bulb yield $\mathrm{kg} /$ plot under field conditions during seasons $2013 / 2014$ and 2014/2015.

\begin{tabular}{|c|c|c|c|c|c|c|}
\hline \multicolumn{2}{|c|}{ Treatments } & \multirow[b]{2}{*}{ Conc. } & \multicolumn{4}{|c|}{ Onion bulb yield kg/plot $\left(10.5 \mathrm{~m}^{2}\right)$} \\
\hline $\begin{array}{c}\text { Bio- } \\
\text { agent }\end{array}$ & Plant oils & & $2013 / 2014$ & Increase \% & $2014 / 2015$ & Increase \% \\
\hline \multirow{10}{*}{ 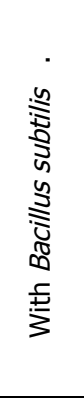 } & \multirow{3}{*}{ Thyme } & 0.5 & 27.2 & 8.0 & 21.9 & 6.8 \\
\hline & & 1.0 & 30.6 & 11.4 & 25.6 & 10.5 \\
\hline & & 2.0 & 31.1 & 11.9 & 26.7 & 11.6 \\
\hline & \multirow{3}{*}{ Cumin } & 0.5 & 30.1 & 10.9 & 24.1 & 9.0 \\
\hline & & 1.0 & 32.6 & 13.4 & 27.8 & 12.7 \\
\hline & & 2.0 & 34.1 & 14.9 & 28.6 & 13.5 \\
\hline & \multirow{3}{*}{ Cardamom } & 0.5 & 24.1 & 4.9 & 19.3 & 4.2 \\
\hline & & 1.0 & 29.1 & 9.9 & 23.2 & 8.1 \\
\hline & & 2.0 & 33.6 & 14.4 & 27.6 & 12.5 \\
\hline & B. subtilis & 0.0 & 21.9 & 2.7 & 16.3 & 1.2 \\
\hline \multirow{10}{*}{ 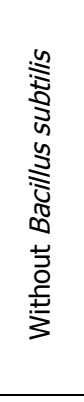 } & \multirow{3}{*}{ Thyme } & 0.5 & 23.2 & 4.0 & 19.1 & 4.0 \\
\hline & & 1.0 & 27.7 & 8.5 & 22.7 & 7.6 \\
\hline & & 2.0 & 32.7 & 13.5 & 24.7 & 9.6 \\
\hline & \multirow{3}{*}{ Cumin } & 0.5 & 27.2 & 8.0 & 21.7 & 6.6 \\
\hline & & 1.0 & 29.5 & 10.3 & 24.8 & 9.7 \\
\hline & & 2.0 & 30.8 & 11.6 & 25.8 & 10.7 \\
\hline & \multirow{3}{*}{ Cardamom } & 0.5 & 22.1 & 2.9 & 17.8 & 2.7 \\
\hline & & 1.0 & 24.8 & 5.6 & 19.9 & 4.8 \\
\hline & & 2.0 & 30.1 & 10.9 & 24.7 & 9.6 \\
\hline & Control & 0.0 & 19.2 & - & 15.1 & - \\
\hline \multicolumn{3}{|c|}{ Fungicides } & 35.4 & 16.2 & 28.2 & 13.1 \\
\hline
\end{tabular}

\begin{tabular}{|c|c|c|c|c|c|c|c|}
\hline LSD. at 5\% & B. subtilis (a) & Plant oils (b) & Conc. (c) & $\mathrm{a} \times \mathrm{b}$ & $\mathrm{a} \times \mathrm{c}$ & $\mathrm{b} \times \mathrm{c}$ & $\mathrm{a} \times \mathrm{b} \times \mathrm{c}$ \\
\hline $2013 / 2014$ & 0.87 & 1.28 & 1.01 & 1.98 & 1.56 & 2.10 & N.S. \\
\hline $2014 / 2015$ & 0.98 & 1.43 & 1.18 & 2.23 & 1.96 & 2.43 & N.S. \\
\hline
\end{tabular}

\section{DISCUSSION}

In the present study, using Bacillus subtilis when applied as soil treatment in combinations with different concentrations of plant oils as transplant dipping treatment were effective in reducing incidence of white rot under artificial and natural infections in greenhouse and field trials, compared with untreated control. This is in agreement with various investigators (El-Moshtohory, 2007, Mahmoud, 2014, and Mahmoud and Gomaa, 2015), who reported that, B. subtilis and essential plant oils play an important role in reducing many plant diseases.

Bacillus subtilis is one of the plant growth-promoting rhizobacteria (PGPR), which suppresses a variety of root and vascular disease caused by soilborne pathogens (Mishra et al., 2013). Awais et al. (2010) stated that the biocontrol activity 
had been associated with the production of certain metabolites such as enzymes, phenazines, bio-compounds, indole derivatives and peptide antibiotic, such as difficidins, bacillomycins and bacillaenes.

Essential oils are natural, complex, aromatic, oily liquids and composed mainly of terpenes, in addition to some other non-terpene components. They have been shown to possess antibacterial, antifungal, antiviral, insecticidal, and antioxidant properties (Coutinho et al., 2010). Essential oils have been known to possess antimicrobial activity by their action through the disruption of the cell membrane (Lixandru et al., 2010). Moreover, Burt (2004) illustrated that, the hydrophobicity of essential oils enables them to affect the lipids of the cell membrane and mitochondria, rendering them permeable and leading to leakage of cell contents. On the other hand, inhibitory action of natural products on moulds involves cytoplasm granulation, cytoplasmic membrane rupture and inactivation and/or inhibition of intercellular and extracellular enzymes. These biological events could take place separately or concomitantly culminating with the inhibition of mycelium growth or spore germination (Abdolmaleki et al., 2008). Generally, in many cases, the antifungal activity of the essential oils results from the interaction between the different classes of compounds such as phenols, aldehydes, ketones, alcohols, esters, ethers or hydrocarbons present in these oils. Several studies have found that a number of these compounds exhibited significant antifungal properties when tested separately (Nestor Bassole and Juliani, 2012).

This was clearly demonstrated with our results, which indicated that, linear growth of the pathogen was significantly reduced by most tested plant oils treatments compared with control. This might be attributed to the content of essential oils constituents with antimicrobial activity such as, tannins, glycosides, and resins, which can be found in certain spices such as cumic aldehyde and pyridine in cumin, 1,8cineol and phenol 1- methylethyl in thyme oil and 1,8-cineole and terpinyl acetete in cardamom which showed clearly in GC-MASS analysis. This in agreement with Segvi'c Klari'c et al., (2007) who showed that p-cymene, and 1,8-cineole were the main components of thyme oil. While, the major components in cardamom oil were 1,8 cineole and terpinyl acetate (El-Moshtohory, 2007). Asghari-Marjanlo et al., (2009) also reported p-cymene $(12.9 \%)$ and cumin aldehyde $(24.9 \%)$ to be the major components of cumin oil which have antifungal activity, enabling them to provide protection of fruit and vegetables against quantitative and qualitative losses, thereby enhancing their shelf-life (Kedia et al., 2014).

The results also indicated that the tested plant oils at different concentrations significantly reduced onion white rot as well increasing of plant growth of (fresh and 
dry) bulb yield grown under greenhouse and field conditions, compared to nontreated control. Data also showed that, there was a relation between plant oil concentrations and their effect on the pathogen and disease. Data clearly indicated that, increasing the concentration of plant oils led to increasing their effect in reducing the incidence of the studied disease. This is agreement with many investigators (ElMoshtohory, 2007, and Mahmoud et al., 2013).

\section{REFERENCES}

1. Abdolmaleki M., Salari M. , Bahraminejad S., Panjeke N. , Abbasi S. 2008. Antifungal effects of Cinnamomum zeylanicum extract on growth of Rhizoctonia solani, Phytophthora oxysporum, Fusarium drechsleri, Bipolaris sorokiniana . plant pathol . 44:255-261

2. Akash K., B. Prakash, K. Prashant and N.K. Dubey 2014. Antifungal and antiaflatoxigenic properties of Cuminum cyminum (L.) seed essential oil and its efficacy as a preservative in stored commodities. Inter. J. Food Microbiol. 3: 1-7

3. Asghari-Marjanlo, A., Mostofi, Y., Shoeibi, S.H., Fattahi, M., 2009. Effect of cumin essential oil on postharvest decay and some quality factors of strawberry. J. Med. PI. 8, 25-43.

4. Awais M., A. Pervez, Asim Yaqub and M.M. Shah, 2010. Production of antimicrobial metabolites by Bacillus subtilis immobilized in polyacrylamide gel Pakistan J. Zool.. 42(3): 267-275

5. Basyony, A.E.; A. H. Ashub, and F. A. Epad, 1989. .Effect of plant population and nitrogen levels on rapeseed oil quality and quantity. Annal Agric. Sci. Moshtohor, 27:761-770.

6. Burt S. 2004. Essential oils: their antibacterial properties and potential applications in foods - a review. Int. J. Food Microbiol.; 94(3):223-253.

7. Callan, N. W.; D. E. Mather and J. B. Miller, 1990. Biopriming seed treatment for biological control of Pythium ultimum pre-emergence damping-off in sh 2 sweet corn. Plant Dis., 74: 368-372.

8. Cook, R.J. 1993. Making greater use of introduced microorganisms for biological control of plant pathogens Annu. Rev. Phytopathol., 31: 53-80.

9. Coutinho H.D.M., Matias E.F.F., Santos K.K.A., Tintino S.R., Souza C.E.S., Guedes G.M.M., Santos F.A.D., Costa J.G.M., Falcao-Silva V.S., and J.P. Siqueira-Junior 2010. Enhancement of the norfloxacin antibiotic activity by gaseous contact with the essential oil of Croton zehntneri. Pharmacognosy.;2(4):362-364. 
10. EL-Bastawesy, A. M. and R. H. Mohamed, 2005. Evaluation of Thyme oil role as antimicrobial, anticarcenogenic and anti-inflammatory agents. Egypt. J. Agric. Res., 83 (2):789-809.

11. El-Hadidy, A.M., 2003. New approaches for controlling some soil borne fungal pathogens on pepper in reclaimed soil. Ph.D. Thesis, Fac. Agric. Ain Shams Univ., $169 \mathrm{pp}$

12. El-Moshtohory, T. A., 2007. Chemical and microbiological studies on some essential oils. Ph.D. Thesis, Fac. of Agric. Moshtohor, Benha Univ. pp

13. Hovius, M. H. Y. and I. L. Goldman. 2004. Evaluation of long-day onions for resistance to white rot infection using greenhouse and laboratory techniques. J. Amer. Soc. Hort. Sci., 129(2):258-265.

14. Jones, A. 2010. Onion White Rot. Grow Your Own Magazine - The Grapevine Forum. Retrieved 2010- 07-22. http://gardener.wikia.com/wiki/Onion white rot

15. Kapoor I.P.S.; B. Singh, G. Singh, V. B. Isidorov, and L. Szczepaniak 2008. Chemistry, antifungal and antioxidant activities of cardamom (Amomum subulatum) essential oil and oleoresins. International Journal of Essential Oil Therapeutics 2: 29-40

16. Kedia, A., Prakash, B., Mishra, P.K., Dubey, N.K., 2014. Antifungal and antiaflatoxigenic properties of Cuminum cyminum (L.) seed essential oil and its efficacy as a preservative in stored commodities. Int. J. Food Microbiol. 168-169, $1-7$.

17. Khalifa, M. M. A., Noher, A. Mahmoud and N. M., Abou-Zeid. 2013 a. Performance of some biofungicides on the most onion economic diseases compared to recommended fungicide in Egypt I- White rot disease control and economical feasibility. Egypt .J. of Appl. Sci., 28 (1): 40-65

18. Khalifa M. M. A., Mahmoud, E.Y., and M.M. Ibrahim 2013 b. Integration of Mycorrhiza and calcium treatments for suppression of Onion white rot disease. Research Journal of Agriculture and Biological Sciences, 8 (4): 385-398.

19. Leal, P.F., M.E. Braga, D.N. Sat, J.E. Carvalho, M.O. Marques and M.A. Meireles, 2003. Functional properties of spice extracts obtained via supercritical fluid extraction. J. Agric.Food Chem., 51 (9): 2520-2525.

20. Lixandru B.E., Dracea N.O., Dragomirescu C.C., Dragulescu E.C., Coldea I.L., Anton L., Dobre E., Rovinaru C., and I. Codita 2010. Antimicrobial activity of plant essential oils against bacterial and fungal species involved in food poisoining and/or food decay. Roum Arch Microbiol Immunol;69(4):224-230. 
21. Mahmoud, E.Y., 2014. Performance of some antagonistic bacteria in minimizing occurrence of peanut damping - off, root and pod rot diseases. Egypt j. Phytopathol. 42 (1):205-220.

22. Mahmoud, E.Y., and Ahmed, M. Gomaa, 2015. Impact of some essential plant oils for controlling of peanut pod rots diseases and aflatoxin. J. Biol. Chem. \& Envirom. Sci., 10 (1): 261-280.

23. Mahmoud, E.Y., Ibrahim, M. M., and T. A. A. Essa, 2013. Efficacy of plant essential oils in controlling damping-off and root rots diseases of peanut as fungicides alternative. Journal of Applied Sciences Research, 9(3): 1612-1622.

24. Mishra D.S., A. Kumar, S. E. Prajapati, A. K. Singh, and S. D. Sharma 2013. Identification of the compatible bacterial and fungal isolates and their effectiveness against plant diseases. Journal of Environmental Biology, 34 (3):183-189.

25. Mohamed, H. A. 2012. Integrated control of onion white rot disease. M. Sc. Thesis Faculty of Agriculture, Moshtohor Benha Univ, 134 pp.

26. Nestor Bassole, H., Juliani, R., 2012. Essential oils in combination and their antimicrobial properties. Molecules 17, 3989-4006, http://dx.doi.org/10. 3390/molecules17043989.

27. Ozcan, M. and O. Erkmen, 2001. Antimicrobial activity of essential oils of Turkish plant spices. Euro. Food Res.Technol., 212(6):658-660.

28. SAS Institute, Inc., 1996. SAS / STAT Users Guide, Version 6, $12^{\text {th }}$ Ed. Volume 2, 846 pp. SAS Institute, Inc. Cary, North Carolina.

29. Segvi 'c Klari' c, M., Kosalec, I., Masteli' c, J., Pieckova, E., Pepeljnak, S., 2007. Antifungal activity of thyme (Thymus vulgaris L.) essential oil and thymol against moulds from damp dwellings. Lett. Appl. Microbiol. 44, 36-42. 


\section{تكامل Bacillus subtilis مع بعض الزيوت \\ النباتية في مكافحة العفن الابيض في البصل بعل فئل}

\section{عماد الدين يوسف محمود - محمد محمود أحمد إبراهيم - فاطمة عبد المطلب مصطفي - زينب نصر الدين حسين}

$$
\text { معهُ بحوث أمراض النباتات - مركز البحوث الزراعية - الجيزة - مصر. }
$$

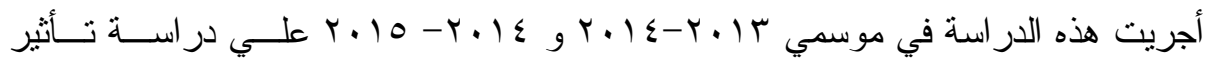

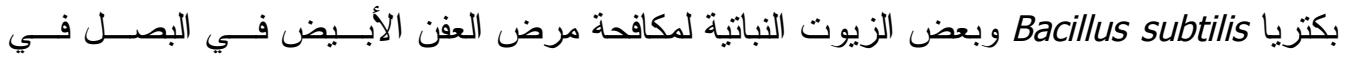

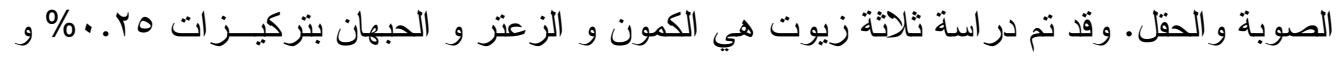

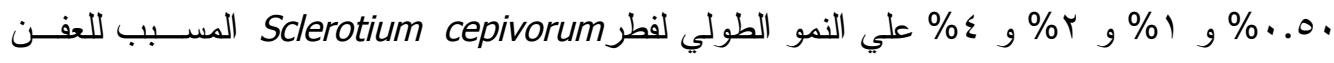
الأبيض في البصل وقد أوضحت النتائج أن زيت الكمون أعطي اعلي تنبيط للفطر يليه زيت الزعتر .

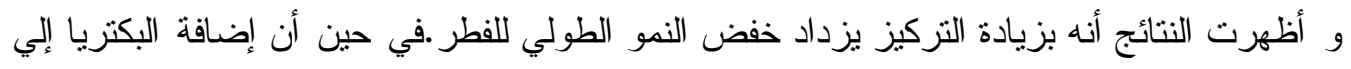

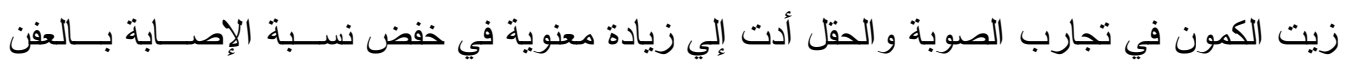

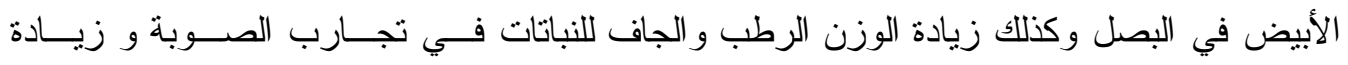

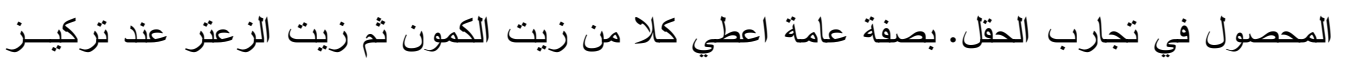
r\% مع إضافة البكتريا أعلى كفاءة في خفض نسبة الإصابة بالمرض بالمقارنة بباقي المعساملات

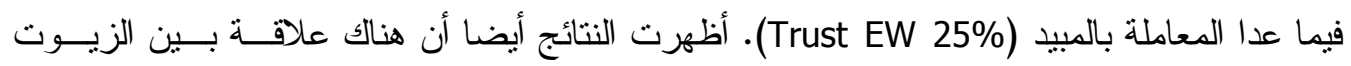

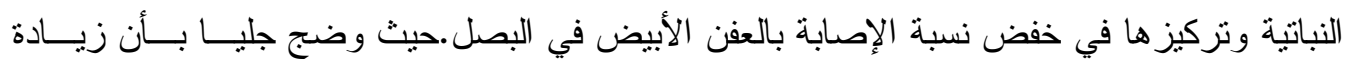

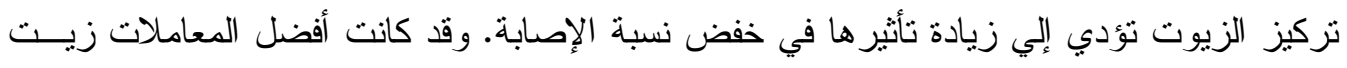

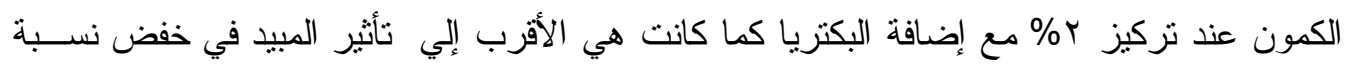

